\title{
DOWNLINK AND UPLINK CAPACITY ENHANCEMENT IN POWER CONTROLLED CELLULAR SYSTEMS
}

\author{
F. Rashid-Farrokhi, K. J. R. Liu, and L. Tassiulas \\ Electrical Engineering Department and Institute for Systems Research \\ University of Maryland at College Park \\ College Park, Maryland 20742
}

\section{ABSTRACT}

The joint power control and base station assignment for downlink is considered in this paper. We present an algorithm that finds a feasible joint base assignment and power control in the downlink which uses the base assignment that is computed by the joint algorithm in the uplink. We show that under some conditions the algorithm also minimizes the sum of downlink powers. Interestingly, there is not always a Pareto optimal power vector for the joint problem in the downlink as is the case in the uplink. This fact is demonstrated through a counter example.

\section{INTRODUCTION}

The objective of power control in wireless networks is to minimize transmitted powers subject to maintaining the link quality by keeping the signal to noise ratio (SNR) above a threshold called minimum protection ratio. The power allocation and base station assignment can be integrated to attain higher capacity and achieve smaller allocated powers. In a network with power control capability, as the mobiles move or new calls arrive, the reassignment of mobiles to base stations helps to find a feasible power allocation and provides a framework for handoff.

In [1], [2] the combined base station assignment and power allocation was proposed for uplink. In those papers, algorithms were proposed to achieve the optimal base assignment and power allocation such that the mobile powers are minimal among all feasible assignments. In [3] it has been observed that for fixed assignment the feasibility of uplink and downlink are equivalent. In this paper we consider the joint power control and base station assignment for downlink. It is interesting to note that unlike the fixed assignment, in the joint problem the uplink and downlink are substantially different. While in the uplink there is a Pareto optimal solution for the

This work is supported in part by the NSF NYI Award MIP9457397, CAREER award NCR-9502614, and AFOSR 95-10061 . power control and base station assignment, this is not the case for the downlink. We will demonstrate this fact through a counter example. Regarding the joint problem in the downlink we will show that the sequence of base station assignment for the joint uplink power control and base station assignment converges to a feasible assignment for the downlink. We use the same iterations to update the downlink power, and we will prove that this algorithm converges to a feasible downlink power allocation and base station assignment when there exists any. We will also show that when the interference is only due to thermal noise and cochannels, our algorithm also minimizes the sum of downlink powers among all feasible assignments. Our proposed algorithm can also be generalized for the networks where uplink and downlink gains are different.

The organization of the paper is as follows: In section III the system model for uplink power control and base station assignment is presented. In section IV we illustrate the differences of the joint problem in the uplink and downlink through an example, and we propose an algorithm to find a feasible solution for the joint problem in the downlink. In section $\mathrm{V}$ we discuss the case where the uplink and downlink gains are different, and finally the performance of our algorithm is evaluated through numerical simulation in section VI.

\section{UPLINK POWER CONTROL AND BASE STATION ASSIGNMENT}

First we consider the power control problem for fixed assignment of mobiles to base stations. Let mobile $i$ be assigned to base station $i$. The aim of power control is to minimize transmitted power such that the SNR at each base station (denoted by $\Gamma_{i}$ ) is no less than a threshold $\gamma_{i}$. That is,

$$
\Gamma_{i}=\frac{G_{i i} P_{i}}{\sum_{j \neq i} G_{j i} P_{j}+N_{i}} \geq \gamma_{i}, \quad i=1, \ldots, M
$$

where $M$ is the number of mobiles, $G_{j i}$ is the link gain between mobile $j$ and base $i . P_{i}$ is the $i$ th mobile power 
and $N_{i}$ is the noise power at the $i$ th base station. The above constraint in matrix form is given by

$$
\mathbf{P} \geq \mathbf{D F P}+\mathbf{u},
$$

where $\mathbf{P}=\left[P_{1}, \ldots, P_{M}\right]^{T}, \mathbf{D}=\operatorname{diag}\left\{\frac{\gamma_{1}}{G_{11}}, \ldots, \frac{\gamma_{M}}{G_{M M}}\right\}$, $\mathbf{u}$ is an element-wise positive vector whose elements are defined as $u_{i}=\gamma_{i} N_{i} / G_{i i}$, and

$$
[\mathbf{F}]_{i j}=\left\{\begin{array}{lll}
0 & \text { if } & j=i \\
G_{j i} & \text { if } & j \neq i
\end{array} .\right.
$$

The power control problem is defined as

$$
\begin{aligned}
& \min \sum_{i} P_{i}, \\
& \text { subject to } \Gamma_{i}>\gamma_{i} .
\end{aligned}
$$

If the spectral radius of $\mathbf{D F}$ is less than unity, i.e., $\rho(\mathbf{D F})<1,[I-\mathbf{D F}]$ is invertible and positive [5]. In this case the network is called feasible and the optimal solution to the power control problem is given by [6]

$$
\widehat{\mathbf{P}}=[I-\mathbf{D F}]^{-1} \mathbf{u} .
$$

It is interesting to note that this solution is Pareto optimal. That is, $\mathbf{P}$ is an elementwise minimal vector among all feasible solutions. The SNR at the mobile $\left(\tilde{\Gamma}_{i}\right)$ is given by

$$
\tilde{\Gamma}_{i}=\frac{G_{i i} \tilde{P}_{i}}{\sum_{j \neq i} G_{i j} \tilde{P}_{j}+\tilde{N}_{i}},
$$

where $\tilde{N}_{i}$ is the noise power at the $i$ th mobile. The $i$ th base station power is denoted by $\tilde{P}_{i}$. The quality constraint $\left(\tilde{\Gamma}_{i}>\gamma_{i}\right)$ can be written in matrix form as $\tilde{\mathbf{P}} \geq \mathbf{D F}^{T} \tilde{\mathbf{P}}+\tilde{\mathbf{u}}$, where $\tilde{\mathbf{P}}=\left[\tilde{P}_{1}, \ldots, \tilde{P}_{M}\right]^{T}$, and $\tilde{u}_{i}=$ $\gamma_{i} \tilde{N}_{i} / G_{i i}$. Similar to uplink, it can be shown that if $\rho\left(\mathbf{D F}^{T}\right)<1$, the optimal downlink power is given by $\widehat{\tilde{\mathbf{P}}}=\left[I-\mathbf{D F}^{T}\right]^{-1} \tilde{\mathbf{u}}$. In [3] it has been shown that the spectral radius of $\mathbf{D F}$ and $\mathbf{D F} \mathbf{F}^{T}$ and consequently the feasibility of uplink and downlink power control with fixed assignment are the same. In a feasible network the optimal power vector can be calculated by a distributed algorithm [4]. The $n$th iteration of this algorithm is given by

$$
P_{i}^{n+1}=\sum_{j \neq k} \frac{\gamma_{i} G_{j k}}{G_{i k}} P_{j}^{n}+\frac{\gamma_{i} N_{k}}{G_{i k}}
$$

The above algorithm can be implemented locally since the right hand side is a function of the total interference received at the $i$ th base station at the $n$th iteration. In matrix form (1) can be written as

$$
\mathbf{P}^{n+1}=\mathbf{D F P}^{n}+\mathbf{u},
$$

Similarly the downlink power can be updated by

$$
\tilde{\mathbf{P}}^{n+1}=\mathbf{D F}^{T} \tilde{\mathbf{P}}^{n}+\tilde{\mathbf{u}},
$$

where $\mathbf{P}^{n}=\left[P_{1}^{n}, \ldots, P_{M}^{n}\right]^{T}$ and $\tilde{\mathbf{P}}^{n}=\left[\tilde{P}_{M}^{n}, \ldots, \tilde{P}_{M}^{n}\right]^{T}$.

In the optimal uplink power control and base station assignment, the objective is to determine the assignment of users to base stations which minimizes the allocated mobile powers. Iterative algorithms that compute the optimal joint base station and power assignment were proposed in [1], [2]. The power update in this case is given by:

$$
P_{i}^{n+1}=\arg \min _{k \in B_{i}}\left\{\sum_{j \neq k} \frac{\gamma_{i} G_{j k}}{G_{i k}} P_{j}^{n}+\frac{\gamma_{i} N_{k}}{G_{i k}}\right\},
$$

where $B_{i}$ is the set of allowable base stations for the $i$ th mobile. A network is feasible if there exists an assignment and power allocation such that the quality constraint is satisfied for each link. In [1], [2] it has been shown that in a feasible network, the above iteration converges to the Pareto optimal power allocation and base station assignment, such that the mobile power is minimal among all feasible base station assignments. In uplink the interference, induced by a certain transmitter to other links, is not affected by base assignment. As a result, there is an assignment that minimizes the transmitted power for all mobiles, while by changing the assignment in the downlink, the locations of transmitters are changed and the interference is changed as well. This feature is the source of fundamental difference of uplink and downlink.

\section{DOWNLINK POWER CONTROL AND BASE STATION ASSIGNMENT}

We enhance the joint power allocation and base station assignment by the following algorithm. We let the uplink select the base station and at the same time we run one power control iteration for downlink using the same assignment. The algorithm steps at the $n$th iteration are as follows:

\section{Algorithm BUD}

1. (B) The base station assignment, denoted by $b_{i}^{n}$, is found to minimize the uplink power:

$$
b_{i}^{n}=\arg \min _{k \in B_{i}}\left\{\sum_{j \neq i} \frac{\gamma_{i} G_{j k}}{G_{i k}} P_{j}^{n}+\frac{\gamma_{i} N_{k}}{G_{i k}}\right\}
$$

2. (U) The uplink power is updated based on the assignment $b_{i}^{n}$ :

$$
P_{i}^{n+1}=\sum_{j \neq i} \frac{\gamma_{i} G_{j b_{i}^{n}}}{G_{i b_{i}^{n}}} P_{j}^{n}+\frac{\gamma_{i} N_{i}}{G_{i b_{i}^{n}}} .
$$


3. (D) The downlink power is updated based on the same assignment $\left(b_{i}^{n}\right)$ :

$$
\tilde{P}_{i}^{n+1}=\sum_{j \neq i} \frac{\gamma_{i} G_{i b_{j}^{n}}}{G_{i b_{i}^{n}}} \tilde{P}_{j}^{n}+\frac{\tilde{N}_{i} \gamma_{i}}{G_{i b_{i}^{n}}} .
$$

The sequences $P_{i}^{n}$ and $b_{i}^{n}$ converge to the optimal power control and base station assignment for the uplink, as it has been shown in [1], [2]. Next we will show the following:

Theorem 1 Algorithm BUD converges to the optimal base station assignment and power allocation for uplink which minimizes each mobile power, and converges to a feasible base station assignment and power allocation for downlink.

Proof: In [1] it has been proved that for any starting power allocation there exists an $N \geq 0$ such that for $n \geq N, b_{i}^{n}=\widehat{b}_{i}$. Consider the positive vectors $\widehat{\mathbf{u}}$ and $\widehat{\tilde{\mathbf{u}}}$ whose elements are defined by $\widehat{u_{i}}=\frac{\gamma_{i} N_{i}}{G \widehat{G}_{i}}$ and $\widehat{\tilde{u}}_{i}=\frac{\gamma_{i} \tilde{N}_{i}}{G \widehat{\hat{i}}_{i}}$. Define $\widehat{\mathbf{D}}=\operatorname{diag}\left\{\frac{\gamma_{1}}{G_{1 b_{1}}}, \ldots, \frac{\gamma_{M}}{G_{M_{M}}}\right\}$, and $\widehat{\mathbf{F}}=\left\{\widehat{F}_{i j}\right\}$,

$$
\widehat{F}_{i j}=\left\{\begin{array}{lll}
0 & \text { if } & j=i \\
G_{\widehat{j b}_{i}} & \text { if } & j \neq i
\end{array} .\right.
$$

For $n>N$ the power control iteration for uplink and downlink can be written in matrix form as

$$
\mathbf{P}^{n}=\widehat{\mathbf{D}} \widehat{\mathbf{F}} \mathbf{P}^{n-1}+\widehat{\mathbf{u}}
$$

and

$$
\tilde{\mathbf{P}}^{n}=\widehat{\mathbf{D}} \widehat{\mathbf{F}}^{T} \tilde{\mathbf{P}}^{n-1}+\widehat{\tilde{\mathbf{u}}} .
$$

If the spectral radius of $\widehat{\mathbf{D}} \widehat{\mathbf{F}}$ is less than unity the above iteration converges to [4]

$$
\widehat{\mathbf{P}}=[I-\widehat{\mathbf{D}} \widehat{\mathbf{F}}]^{-1} \widehat{\mathbf{u}} \text {. }
$$

Since $\rho(\widehat{\mathbf{D}} \widehat{\mathbf{F}})=\rho\left(\widehat{\mathbf{D}} \widehat{\mathbf{F}}^{T}\right)$, the optimal assignment for uplink is feasible for downlink as well, and the downlink power iterations converge to

$$
\widehat{\tilde{\mathbf{P}}}=\left[I-\widehat{\mathbf{D}} \widehat{\mathbf{F}}^{T}\right]^{-1} \widehat{\tilde{\mathbf{u}}} .
$$

Our algorithm finds a feasible assignment for downlink if there is any, and as we will see later, in general there is no assignment that minimizes the downlink power vector element-wise. However, in the sequel we will prove that in a special case that $N_{i}$ is the same for all base stations and $\tilde{N}_{i}$ is also the same for all mobiles, e.g., when $N_{i}$ and $\tilde{N}_{i}$ are solely due to thermal noise, the sum of base station powers is also minimal among all feasible base station assignments.
Theorem 2 In the case that $N_{i}=N$ and $\tilde{N}_{i}=\tilde{N}$, in a feasible network algorithm BUD also minimizes $\sum_{i} \tilde{P}_{i}$.

Proof: Since the network is feasible the spectral radius of $\widehat{\mathbf{D}} \widehat{\mathbf{F}}$ and $\widehat{\mathbf{D}} \widehat{\mathbf{F}}^{T}$ are less than unity. In this case (5) and (6) are given by [5]

$$
\widehat{\mathbf{P}}=\sum_{k=0}^{\infty}(\widehat{\mathbf{D}} \widehat{\mathbf{F}})^{k} \mathbf{u}
$$

and

$$
\widehat{\tilde{\mathbf{P}}}=\sum_{k=0}^{\infty}\left(\widehat{\mathbf{D}} \widehat{\mathbf{F}}^{T}\right)^{k} \widehat{\mathbf{u}} .
$$

It can be shown that $\widehat{\mathbf{u}}=N \widehat{\mathrm{D}} \mathbf{1}$ and $\widehat{\tilde{\mathbf{u}}}=\tilde{N} \widehat{\mathbf{D}} \mathbf{1}$, where $\mathbf{1}$ is the all one vector. Therefore the optimal power vectors for downlink and uplink are given by

$$
\widehat{\mathbf{P}}=N \sum_{k=0}^{\infty}(\widehat{\mathbf{D}} \widehat{\mathbf{F}})^{k} \widehat{\mathbf{D}} \mathbf{1},
$$

and

$$
\widehat{\tilde{\mathbf{P}}}=\tilde{N} \sum_{k=0}^{\infty}\left(\widehat{\mathbf{D}} \widehat{\mathbf{F}}^{T}\right)^{k} \widehat{\mathbf{D}} \mathbf{1}
$$

respectively. The sum of mobile powers (uplink) is given by

$$
\mathbf{1}^{T} \widehat{\mathbf{P}}=N \sum_{k=0}^{\infty} \mathbf{1}^{T}(\widehat{\mathbf{D}} \widehat{\mathbf{F}})^{k} \widehat{\mathbf{D}} \mathbf{1}
$$

and the sum of base station powers (downlink) is given by

$$
\mathbf{1}^{T} \widehat{\tilde{\mathbf{P}}}=\tilde{N} \sum_{k=0}^{\infty} \mathbf{1}^{T}\left(\widehat{\mathbf{D}} \widehat{\mathbf{F}}^{T}\right)^{k} \widehat{\mathbf{D}} \mathbf{1}
$$

Since (8) is a scalar it is not changed by transposing, i.e.,

$$
\begin{aligned}
\mathbf{1}^{T} \hat{\tilde{\mathbf{P}}} & =(\hat{\tilde{\mathbf{P}}})^{T} \mathbf{1}=\tilde{N} \sum_{k=0}^{\infty} \mathbf{1}^{T} \widehat{\mathbf{D}}(\widehat{\mathbf{F}} \hat{\mathbf{D}})^{k} \mathbf{1} \\
& =\frac{\tilde{N}}{N} N \sum_{k=0}^{\infty} \mathbf{1}^{T}(\hat{\mathbf{D}} \widehat{\mathbf{F}})^{k} \hat{\mathbf{D}} \mathbf{1}=\frac{\tilde{N}}{N} \mathbf{1}^{T} \widehat{\mathbf{P}}
\end{aligned}
$$

The optimal base station assignment and power control achieves the minimal power vector among all feasible assignments, and the sum of uplink and downlink powers are the same up to a constant. Therefore, the sum of powers for downlink is also minimal among all feasible assignments.

In the following we show that, in general, there is no pointwise optimal solution to the joint power control and base station assignment in the downlink. Consider a downlink network of two mobiles and three base stations 
as illustrated in Fig. 1. We consider two assignments of base stations to mobiles. In the first assignment $\left(a_{1}\right)$, $m_{1}$ is assigned to $B_{1}$ and $m_{2}$ is assigned to $B_{2}$. In the second assignment $\left(a_{2}\right) m_{1}$ is assigned to $B_{1}$ and $m_{2}$ is assigned to $B_{3}$. Let the noise power at all mobiles be $\tilde{N}$ and the SNR threshold be $\gamma$. The minimum power allocation which satisfies the link quality is given by

$$
\tilde{\mathbf{P}}^{a_{1}}=\left[I-\gamma \mathbf{D}^{a_{1}} \mathbf{F}^{a_{1}}\right]^{-1} \tilde{\mathbf{u}},
$$

where $\mathbf{D}^{a_{1}} \mathbf{F}^{a_{1}}$ is the gain matrix under assignment $a_{1}$. The base station powers are given by

$$
\tilde{P}_{1}^{a_{1}}=\alpha\left(\frac{\gamma G_{12}}{G_{11} G_{22}}+\frac{1}{G_{11}}\right)
$$

and

$$
\tilde{P}_{2}^{a_{1}}=\alpha\left(\frac{\gamma G_{21}}{G_{11} G_{22}}+\frac{1}{G_{22}}\right)
$$

where $\alpha=\gamma \tilde{N} /\left(1-\frac{\gamma^{2} G_{12} G_{21}}{G_{11} G_{22}}\right)$. In Fig. 1(a) $m_{1}$ is closer to its base station. Therefore, $G_{11}>G_{22}$ and $G_{12}<$ $G_{21}$. In this case $\tilde{P}_{1}^{a_{1}}<\tilde{P}_{2}^{a_{1}}$. Similarly, in Fig. 1(b) the optimal power vectors are given by

$$
\tilde{P}_{1}^{a_{2}}=\beta\left(\frac{\gamma G_{13}}{G_{11} G_{23}}+\frac{1}{G_{11}}\right)
$$

and

$$
\tilde{P}_{2}^{a_{2}}=\beta\left(\frac{\gamma G_{21}}{G_{11} G_{23}}+\frac{1}{G_{23}}\right) .
$$

where $\beta=\gamma \tilde{N} /\left(1-\frac{\gamma^{2} G_{13} G_{21}}{G_{11} G_{23}}\right)$. In this case since $m_{2}$ is closer to its assigned base station, $G_{11}<G_{23}$ and $G_{13}>G_{21}$, i.e., $\tilde{P}_{2}^{a_{2}}<\tilde{P}_{1}^{a_{2}}$. Also if the location of $B_{3}$ is close enough to $m_{2}$ such that $G_{13} / G_{23}>G_{12} / G_{22}$, then $\tilde{P}_{1}^{a_{1}}<\tilde{P}_{1}^{a_{2}}$. That is, by changing the assignment from Fig. $1(\mathrm{a})$ to Fig. $1(\mathrm{~b}), \tilde{P}_{1}$ is increased while $\tilde{P}_{2}$ is decreased, which leads us to this conclusion that in general there is no assignment that minimizes all base station powers among all feasible assignments.

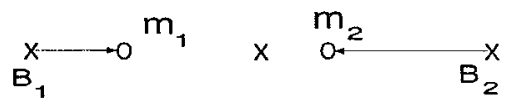

(a)

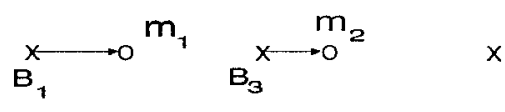

(b)

Figure 1: Feasible assignments for uplink; base stations are shown by $\mathrm{x}$ and mobiles are shown by 0 .

\section{FREQUENCY DIVISION DUPLEX NETWORKS}

In the above analysis we have assumed that the link gain for uplink and downlink are the same. This assumption is valid in Time Division Duplex (TDD) networks or in a Frequency Division Duplex (FDD) network where the uplink and downlink frequencies are close. In FDD networks where the uplink and downlink gains are different, in order to update the uplink powers we use the first two steps of the algorithm BUD (using uplink gains). In this part, the algorithm can be implemented locally, since the base station assignment and power updates are functions of the interference at each base station. The downlink powers are updated by algorithm BUD where all link gains are replaced with the downlink gains. That is, we have virtual uplinks with the same link gains as in the downlink. From the previous section we know that this algorithm converges to a feasible base station assignment which is different from that of uplink. Since the virtual uplink gains are not related to any receiver, the first two steps of the algorithm have to be implemented in a central unit. However, the downlink power update can be implemented locally by measuring the total interference at each mobile. In networks where the downlink and uplink gains differ by a multiplicative factor (due to frequency shift) the feasibility of uplink and downlink gain matrices are the same, i.e., the uplink base station assignment is also feasible for downlink. Therefore in this case both uplink and downlink power allocation and base station assignment can be implemented locally.

\section{SIMULATION RESULTS}

We simulate the performance of the proposed algorithm in a CDMA network. Fig. 2 shows a hexagonal network with 36 base stations, located at the center of each cell. The radius of each cell is unity and the quality constraint requires $\gamma_{0}=0.03$. The link gain is modeled as $G_{i j}=K / d_{i j}^{4}$, where $d_{i j}$ is the distance between $i$ th mobile and $j$ th base station, and $K=6.96 \times 10^{-3}$. The noise power at each receiver is $3.66 \times 10^{-11}$ [7]. 300 users, shown by dots in Fig. 2, are randomly distributed in the area $[-0.86,10.4] \times[-1,8.5]$. In a traditional assignment each user in a cell is assigned to the closest base station (the center of a cell). In a power control and base station assignment the users are assigned to the optimal base stations. The effect of joint base station assignment and power control is more significant in improving the local capacity of a network in a congested area [1]. Therefore, in order to evaluate the performance of our algorithm we have randomly added users to a specific cell in the network, and we have measured the local 
capacity enhancement in the uplink and downlink. For both fixed and dynamic base assignment, in a TDD network, the total transmitted power as a function of the number of users is plotted in Fig. 3. The users inside each polygon are assigned to the same base station by the joint algorithm. Compared to fixed assignment, we can almost double the local capacity of the network in both uplink and downlink. Since the noise power for mobiles and base stations are assumed to be the same, the total power for both uplink and downlink are the same (Fig. 3).

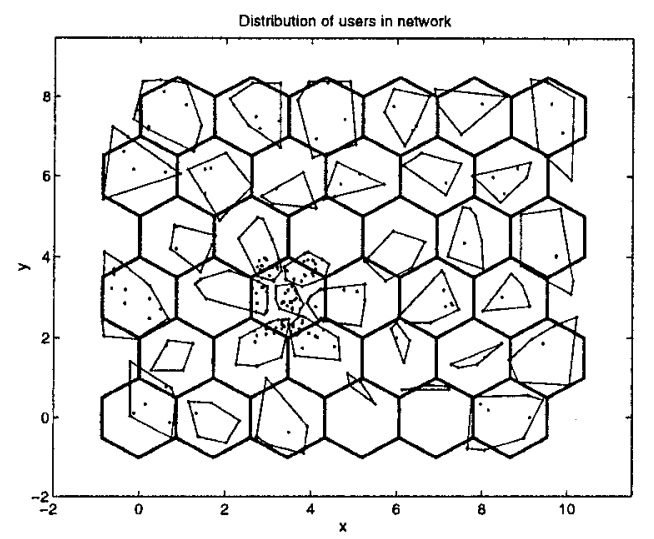

Figure 2: The location of mobiles in a cellular network.

In an FDD network with $10 \%$ difference between uplink and downlink the total sum of mobile and base station powers are plotted in Fig. 4. In the downlink power control and base assignment we consider two scenarios. The solid curve shows the case when in algorithm BUD downlink gains are used in all three steps of the algorithm. The + marks show the result of the simulation when the uplink base station assignment is used for downlink. The total capacity and in fact the assignment for both cases are the same while the first one is implemented locally.

\section{CONCLUSION}

We have stressed the differences of joint power control and base station assignment in uplink and downlink. We have shown that there is no Pareto optimal solution for joint problem in downlink. Therefore, we have proposed an algorithm that finds a feasible solution for the joint problem when there exists any. Moreover, we have proved that in some special cases the solution is also optimal in terms of the sum of base station powers.

\section{REFERENCES}

[1] S. V. Hanly, "An algorithm for combined cell-site selection and power control to maximize cellular

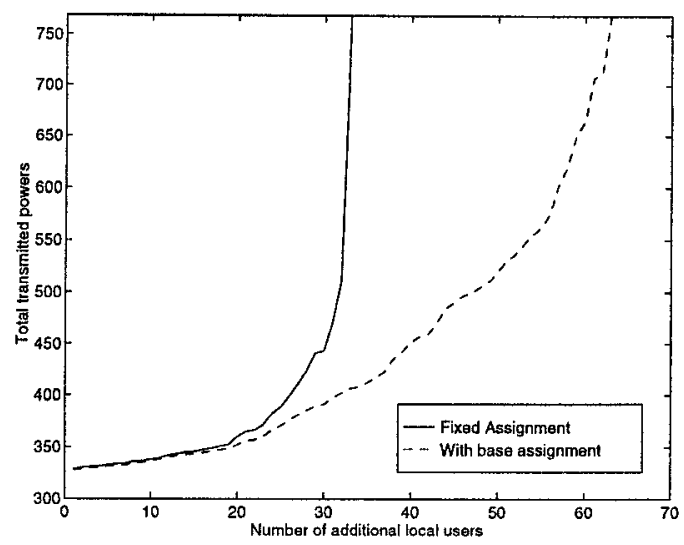

Figure 3: The total transmitted powers versus the number of users in a TDD network.

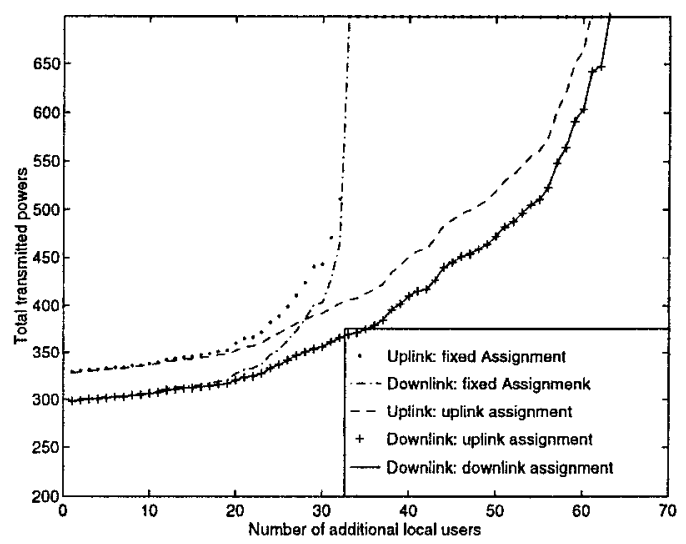

Figure 4: The total transmitted powers versus the number of users in a FDD network.

spread spectrum capacity," IEEE J. Select. Areas Commun., Vol. 13, No. 7, Sept. 1995.

[2] R. Yates and C. Y. Huang, "Integrated power control and base station assignment," IEEE Trans. Vehicular Technol., vol. 44, No. 3, Aug. 1995.

[3] J. Zander and M. Fordigh, Comments on "Performance of optimum transmitter power control in cellular radio systems," IEEE Trans. Vehic. Technol., vol. VT-43, pp. 636, Aug. 1994.

[4] J. Zander, "Distributed cochannel interference control in cellular radio systems," IEEE Trans. Vehicular Technol., vol. 41, Aug. 1992.

[5] F. R. Gantmacher, "The Theory of Matrices," vol. 2, Chelsea, New York, 1990.

[6] D. Mitra, "An asynchronous distributed algorithm for power control in cellular radio systems," in Fourth WINLAB Workshop on Third Generation Wireless Information Networks, 1993.

[7] T. S. Rappaport, "Wireless Communications Principles and Pratice," IEEE Press, New York, 1996. 